M. DI Carlo ${ }^{1}$, C. Ventura ${ }^{2}$, P. Cesaroni ${ }^{1}$, M. Carotti ${ }^{2}$, A. Giovagnoni ${ }^{2}$, F. Salaffi ${ }^{1}$

${ }^{1}$ Università Politecnica delle Marche, Rheumatological Clinic, Jesi, Italy;

${ }^{2}$ Università Politecnica delle Marche, Radiology Department, ANCONA, Italy

Background: The pathophysiology of fibromyalgia syndrome (FM) still needs to be fully clarified. In addition to the central sensitization mechanisms, some evidence suggests an involvement of the peripheral nervous system, mainly intended as small fibers neuropathy. The sural nerve showed some alterations, in terms of increasead cross-sectional area (CSA), in the course of small fibers neuropathy. Objectives: To evaluate sural nerve CSA and factors associated with increased CSA in FM patients.

Methods: A cross sectional evaluation was conducted in consecutive FM patients according to the 2016 American College of Rheumatology criteria. Demographic, clinimetric parameters (in particular the revised Fibromyalgia Impact Questionnaire [FIQR] to assess the severity of the disease and the PainDetect Questionnaire [PDQ] to evaluate neuropathic pain features) and the sural nerve dimensions measured by ultrasound were recorded for each patient. The size of the sural nerve was described in terms of the mean cross-sectional area (CSA) measured bilaterally. CSA was measured at the leg level, $14 \mathrm{~cm}$ from the apex of the lateral malleolus, where the sural nerve is detectable as a structure adjacent to the small saphenous vein in the distal portion of the leg. The ultrasound examination was performed with a MyLab Class C (Esaote S.p.A., Genoa, Italy) equipped with a 6-18 MHz multifrequency broad band probe. CSA was compared with demographic and clinimetric parameters through one-way analysis of variance (ANOVA). A multiple regression was also conducted using CSA as dependent variable, with age, body mass index (BMI), disease duration, FIQR and PDQ as independent variables.

Results: The study involved 110 FM patients (105 women and five men), with a mean age of $50.7( \pm 11.1)$ years and a mean disease duration of $5.8( \pm 5.2)$ years. Sural nerve CSA showed a statistically significant increase in patients with higher PDQ scores $(p=0.0096)$ and, even more significantly, in overweight or obese subjects $(p<0.001)$. The multiple regression analysis, using CSA as dependent variable, confirmed that the PDQ score $(p=0.0049)$ and the body mass index $(p<0.0001)$ are the only two independent variables associated with CSA size (Table 1).

Table 1. Multiple regression analysis of the independent variables related to the mean cross-sectional area (dependent variable) of sural nerve.

\begin{tabular}{|c|c|c|c|c|c|c|}
\hline Indipendent variables & Coefficient & Standard error & $t$ & $\mathrm{p}$ & $r_{\text {partial }}$ & $r_{\text {semipartial }}$ \\
\hline Constant & -0.2551 & & & & & \\
\hline BMI & 0.07592 & 0.01847 & 4.111 & 0.0001 & 0.3739 & 0.3462 \\
\hline Disease duration & -0.02492 & 0.02092 & -1.191 & 0.2364 & -0.1160 & 0.1003 \\
\hline Age & 0.002455 & 0.009833 & 0.250 & 0.8033 & 0.02448 & 0.02103 \\
\hline FIQR & 0.0001376 & 0.006862 & 0.0201 & 0.9840 & 0.001966 & 0.001689 \\
\hline PDQ & 0.05272 & 0.01997 & 2.639 & 0.0096 & 0.2506 & 0.2223 \\
\hline
\end{tabular}

Abbreviations: $\mathrm{BMI}=$ Body Mass Index; FIQR=revised Fibromyalgia Impact Questionnaire; $\mathrm{PDQ}=$ PainDetect Questionnaire

Conclusion: Increased sural nerve CSA is associated with neuropathic like pain features and BMI. Overweight and obesity appear to be associated with an FM phenotype with greater peripheral involvement than normal-weight subjects. Sural nerve ultrasound, an easy to perform examination, could be a useful tool to identify this kind of patients.

References:

[1] Ebadi H, Siddiqui H, Ebadi S, Ngo M, Breiner A, Bril V. Peripheral Nerve Ultrasound in Small Fiber Polyneuropathy. Ultrasound Med Biol. 2015;41(11):2820-6. doi: 10.1016/j.ultrasmedbio.2015.06.011

[2] Giannoccaro MP, Donadio V, Incensi A, Avoni P, Liguori R. Small nerve fiber involvement in patients referred for fibromyalgia. Muscle Nerve. 2014;49(5):757-9. doi: 10.1002/mus.24156

Disclosure of Interests: : None declared

DOI: 10.1136/annrheumdis-2020-eular.2461

\section{THU0478 IS NEUROPATHIC PAIN IN INFLAMMATORY RHEUMATIC DISORDERS AN UNDERESTIMATED PROBLEM? RESULTS FROM THE GERMAN PAINDETECT DATABASE}

P. Sewerin ${ }^{1}$, R. Freynhagen ${ }^{2}$, T. Tölle ${ }^{3}$, M. Hammer ${ }^{4}$, C. Baerwald ${ }^{5}$, J. Walter ${ }^{6}$ R. Schröder ${ }^{7}$, M. Schneider ${ }^{1}$, R. Baron ${ }^{8} .{ }^{1}$ Heinrich-Heine-University Duesseldorf, Department and Hiller Research Unit for Rheumatology, Duesseldorf, Germany; ${ }^{2}$ Division of Anaesthesiology, Intensive Care, Pain \& Pallitive Medicine, Benedictus Krankenhaus, Tutzing, Germany; ${ }^{3}$ Department of Neurology, University Hospital TU Munich Rechts der Isar, Munich, Germany; ${ }^{4}$ Department of Rheumatology, Hospital St. Josef-Stift Sendenhorst, Sendenhorst, Germany; ${ }^{5}$ Department of Rheumatology, Division of Endocrinology, Nephrology and Rheumatology, University Hospital Leipzig,
Leipzig, Germany; ${ }^{6}$ Ambulatory Medical Care Center - Rheumatology, Rendsburg, Germany; ${ }^{7}$ Pfizer Pharma GmbH, Berlin, Germany; ${ }^{8}$ Division of Neurological Pain Research and Therapy, Department of Neurology, University Hospital Schleswig-Holstein, Campus Kiel, Kiel, Germany

Background: The aim of the study was to investigate the impact and relevance of neuropathic pain in inflammatory rheumatic disorders (IRD) and osteoarthritis (OA). Objectives: Pain is one of the main symptoms in patients with IRD and OA. To enhance a mechanistic based treatment of pain the differentiation between nociceptive and neuropathic pain via screening tools (e.g. painDETECT questionaire) might possibly be helpful. The goal of the study was to investigate (1) if neuropathic pain is a significant burden for patients with IRD and (2) if pain patterns differs from degenerative joint diseases such as $\mathrm{OA}$ in over 9.000 patients in each group. Methods: painDETECT is a questionnaire that has been evaluated and used in numerous clinical trials to detect neuropathic pain in various diseases. The collected data is centrally managed and evaluated. In total (end of 2019) 395.984 patients have been documented. Out of the painDETECT database 9256 patients with IRD and 9436 patients with OA were extracted, analyzed and compared on their neuropathic pain pattern (screening was performed using the painDETECT-questionaire., $\mathrm{PDQ}$ ). Secondary parameters were: intensity of pain, functional status, depression, chronicity and sleep disorder. Patients had been recruited from general practitioners (GPs), Rheumatologists, Orthopedics and Neurologists from 862 office-based physicians into the painDETECT-database. This project is an open label registry study in Germany.

Results: The median PDQ-score of patients with inflammatory rheumatic disorders adds up to 14,2 (1-38) and of OA patients to $13,8.28 .7 \%$ of inflammatory rheumatic disorders and $27.2 \%$ of OA-patients showed signs for neuropathic pain by positive $P D Q$. The difference was according to this high patient numbers statistically significant $(\mathrm{P}=0.0015)$. VAS-Score, Depressions-Score, Chronicity -Score and Functional -Score showed no clinically relevant differences between these two groups.

Conclusion: Nearly one third of patients with IRD as well as patients with OA showed neuropathic pain components by using PDQ. Despite increasingly better disease control through more effective therapies, pain still remains a major burden for many patients and has a profound impact on their quality of life. The present data indicate a surprisingly high symptoms of neuropathic pain even in IRD patients and should be considered in the management of our patients. A new documentation system for Rheumatologists (RheumaAssist) could help to address these questions.

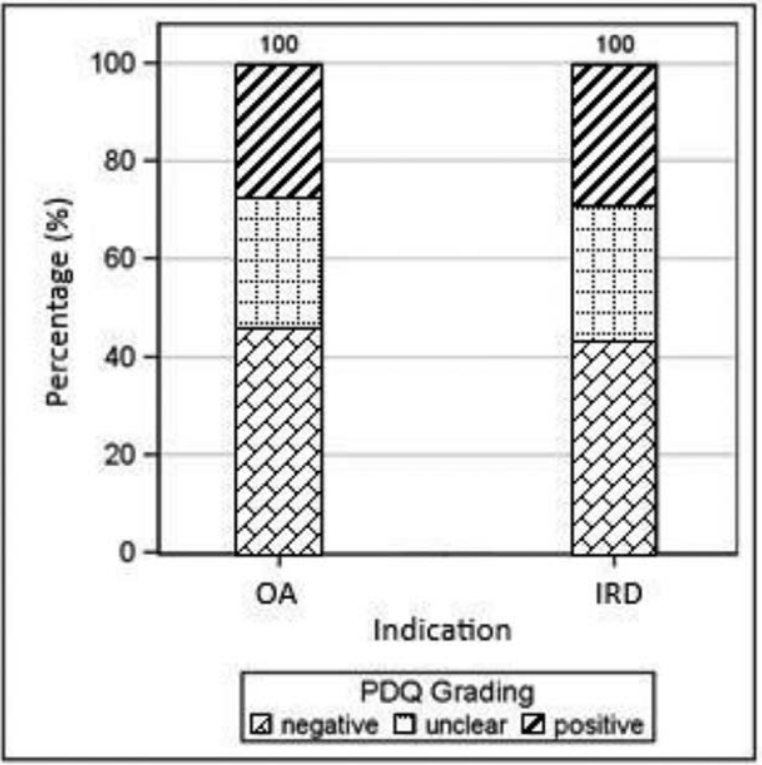

Percentage of PDQ-categories (negative/unclear/positive) for patients with inflammatory rheumatic disorders (IRD) or Arthrosis (OA)

Acknowledgments: This investigation was supported within a grant of Pfizer Deutschland $\mathrm{GmbH}$.

Disclosure of Interests: : Philipp Sewerin Grant/research support from: AbbVie Deutschland $\mathrm{GmbH} \&$ Co. KG

Bristol-Myers Squibb Celgene $\mathrm{GmbH}$

Lilly Deutschland $\mathrm{GmbH}$

Novartis Pharma GmbH Pfizer Deutschland GmbH

Rheumazentrum Rhein-Ruhr, Consultant of: AMGEN GmbH AbbVie Deutschland $\mathrm{GmbH}$ \& Co. KG Biogen GmbHBristol-Myers Squibb Celgene GmbH Chugai Pharma arketing Ltd. / Chugai Europe GmbHHexal Pharma Janssen-CilagGmbH Johnson \& Johnson Deutschland GmbHLilly Deutschland GmbH / Lilly Europe / Lilly Global Novartis Pharma GmbH Pfizer Deutschland 
GmbH Roche Pharma Rheumazentrum Rhein-Ruhr Sanofi-Genzyme Deutschland $\mathrm{GmbH}$ Swedish Orphan Biovitrum GmbH UCB Pharma $\mathrm{GmbH}$, Speakers bureau: AMGEN GmbH AbbVie Deutschland GmbH \& Co. KG Biogen GmbHBristol-Myers Squibb Celgene $\mathrm{GmbH}$ Chugai Pharma arketing Ltd. / Chugai Europe GmbHHexal Pharma Janssen-CilagGmbH Johnson \& Johnson Deutschland GmbHLilly Deutschland GmbH / Lilly Europe / Lilly Global Novartis Pharma GmbH Pfizer Deutschland GmbH Roche Pharma Rheumazentrum Rhein-Ruhr Sanofi-Genzyme Deutschland GmbH Swedish Orphan Biovitrum GmbH UCB Pharma GmbH, Rainer Freynhagen Consultant of: AOP Orphan Pharma, Grünenthal, Lilly, Merck, Mitsubishi Tanabe Pharma, Pfizer, Scilex Pharmaceutics, Speakers bureau: AOP Orphan Pharma, Grünenthal, Lilly, Merck, Mitsubishi Tanabe Pharma, Pfizer, Scilex Pharmaceutics, Thomas Tölle Consultant of: AOP Orphan, Almiral Hermal, Bionest Partners, Benkitt Renkiser, Grünenthal, Hexal, Indivior, Kaia Health, Lilly, Medscape Mundipharma, MSD, Novartis, Pfizer, Recordati Pharma, Sanofi-Aventis, and TAD Pharma, Speakers bureau: AOP Orphan, Almiral Hermal, Bionest Partners, Benkitt Renkiser, Grünenthal, Hexal, Indivior, Kaia Health, Lilly, Medscape Mundipharma, MSD, Novartis, Pfizer, Recordati Pharma, Sanofi-Aventis, and TAD Pharma, Michael Hammer Consultant of: Abbvie, Pfizer, Medac and Janssen, Speakers bureau: Abbvie, Pfizer, Medac and Janssen, Christoph Baerwald Consultant of: CGB received speaker or consulting fees from AbbVie, Paid instructor for: CGB received speaker or consulting fees from AbbVie, Speakers bureau: CGB received speaker or consulting fees from AbbVie, Jochen Walter Consultant of: Pfizer, Speakers bureau: AbbVie, Frauenhofer Institut, Gilead, Janssen-Cilag, Medac, Novartis, Pfizer, Ralf Schröder Shareholder of: Pfizer Pharma $\mathrm{GmbH}$, Employee of: Pfizer Pharma $\mathrm{GmbH}$, Matthias Schneider Grant/research support from: GSK, UCB, Abbvie, Consultant of: Abbvie, Alexion, Astra Zeneca, BMS, Boehringer Ingelheim, Gilead, Lilly, Sanofi, UCB, Speakers bureau: Abbvie, Astra Zeneca, BMS, Chugai, GSK, Lilly, Pfizer, Sanofi, Ralf Baron Consultant of: RB received speaker or consulting fees from AbbVie, Paid instructor for: RB received speaker or consulting fees from AbbVie, Speakers bureau: RB received speaker or consulting fees from AbbVie

DOI: 10.1136/annrheumdis-2020-eular.4457

\section{THU0479 ASSOCIATION BETWEEN CENTRAL SENSITIZATION AND CLINICAL AND ULTRASONOGRAPHIC PARAMETERS IN INFLAMMATORY ARTHRITIDES}

G. Adami ${ }^{1}$, E. Gerratana ${ }^{2}$, A. Giollo ${ }^{1}$, C. Benini ${ }^{1}$, E. Vantaggiato ${ }^{1}$, D. Rotta ${ }^{1}$, L. Idolazzi ${ }^{1}$, D. Gatti ${ }^{1}$, M. Rossini ${ }^{1}$, A. Fassio ${ }^{1} .{ }^{1}$ University of Verona, Verona, Italy; ${ }^{2}$ University of Messina, Messina, Italy

Background: Central sensitization (CS) is an important feature of patients with chronic pain, especially rheumatoid arthritis (RA) and psoriatic arthritis (PsA) patients. CS might interfere with the clinical evaluation of inflammation. Central Sensitization Inventory (CSI) is a validated instrument for assessing central sensitization.

Objectives: We seek to investigate the inference of central sensitization (assessed with the CSI) on clinical (disease activity scores) and ultrasound parameters (US 7-joints score) in RA and PSA patients.

Methods: We conducted a cross-sectional analysis on patients with an established diagnosis of RA or polyarticular PsA. Demographic, anamnestic and clinical parameters were collected. Disease activity was measured with SDAI in RA patients and with DAPSA in PsA patients. The presence and severity of synovitis was measured with the US 7-joints score. Exclusion criteria included: diagnosis of fibromyalgia, depression and patients with PsA with enthesitis predominant and/or spondylitis subtypes. Differences between variables were analysed with t-test and ANOVA for multiple comparisons. Correlation between continuous variables was analysed with Pearson correlation. CSI was analysed either as positivelnegative (threshold 40 points) or divided in four categories, i.e., subclinical ( $\leq 29)$, mild (30-39), moderate (40-49), severe (50$59)$ and extreme $(\geq 60)$.

Results: We enrolled 42 patients in the study. Descriptive characteristics of the study population are presented in table 1. We found no difference in clinical parameters between diseases, sex or age. Women had a higher CSI score compared to men (mean 39.3 vs $26.7 p=0.005$ ). We found a correlation between CSI score and DAPSA $\left(r^{2} 0.39, p=0.001\right)$, number of tender joints $\left(r^{2} 0.13, p=0.02\right)$ and $\mathrm{HAQ}\left(r^{2} 0.47, \mathrm{p}<0.001\right)$ (Figure 1) while we found no correlation between CSI score and SDAI or other clinical parameters. We found a significant difference in DAPSA, tender joints count and HAQ between CSI categories (ANOVA $p=0.01$, $p=0.02$ and $p<0.001$ respectively). US 7-joints score was associated with SDAI $\left(r^{2}\right.$ $0.33, p=0.03)$, number of swollen joints $\left(r^{2} 0.28, p=0.002\right)$ and disease duration $\left(r^{2} 0.35, p<0.001\right)$ but not with DAPSA or tender joints.
Table 1. Descriptive characteristics of the study population

\begin{tabular}{llc}
\hline Age (mean, SD in years) & & $55.9(12.3)$ \\
\hline BMI & & $25.9(3.9)$ \\
CSI questionnaire score (mean) & & $35(16)$ \\
CSI categories (n, \%) & Subclinical & $18(42.9 \%)$ \\
& Mild & $9(21.4 \%)$ \\
& Moderate & $8(19.0 \%)$ \\
& Severe & $4(9.5 \%)$ \\
Diagnosis (n, \%) & Extreme & $3(7.1 \%)$ \\
& PsA 52.4\% & 9 men 13 women \\
CSI score in PsA (mean, SD) & RA 47.6\% & 2 men 18 women \\
CSI score in RA (mean, SD) & & $34.4(16.5)$ \\
CRP (median, IQR in mg/L) & & $34.8(15.5)$ \\
Tender joints (mean, SD) & & $1.85(1.0-4.6)$ \\
Swollen joints (mean, SD) & & $2(2)$ \\
SDAI categories (n, \%) & Remission & $1(2)$ \\
& Low disease activity & $4(20.0 \%)$ \\
& Moderate disease activity & $8(40.0 \%)$ \\
DAPSA categories (n, \%) & Severe disease activity & $7(35.0 \%)$ \\
& Remission & $1(5.0 \%)$ \\
& Low disease activity & $6(33.3 \%)$ \\
& Moderate disease activity & $4(22.2 \%)$ \\
US 7-joints score (mean, SD) & Severe disease activity & $7(38.9 \%)$ \\
HAQ (mean, SD) & & $1(5.6 \%)$ \\
Prednisone equivalent (mean, SD in mg/day) & $6(7)$ \\
Biological DMARD (n, \%) & No & $0.6(0.6)$ \\
Conventional DMARD (n, \%) & Yes & $1.47(3.2)$ \\
& No & $15(35.7 \%)$ \\
& Yes & $27(64.3 \%)$ \\
& & $13(31.0 \%)$ \\
& & $29(69.0 \%)$ \\
\hline
\end{tabular}

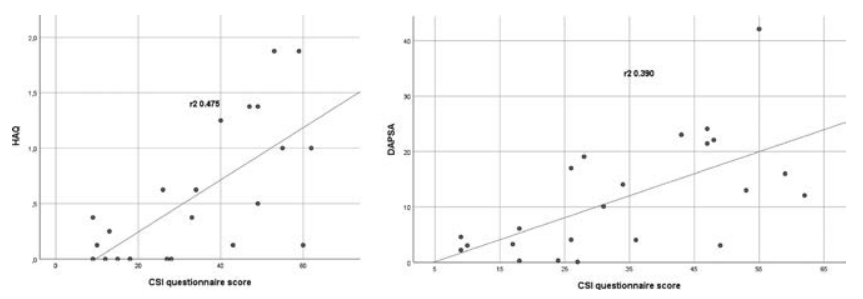

Figure 1. Correlation between DAPSA score and CSI score and between HAQ and CSI score

Conclusion: We found an association between CS and sex, functional disability, tender joints count and disease activity score in PsA patients while there was no correlation between RA disease activity and central sensitization. US 7-joints score was associated with swollen joints count, disease duration and disease activity in RA patients but not in PsA patients. In PsA patients, DAPSA might be more influenced by central sensitization, especially in female individuals.

Disclosure of Interests: Giovanni Adami: None declared, Elisabetta Gerratana: None declared, Alessandro Giollo: None declared, Camilla Benini: None declared, Elisabetta Vantaggiato: None declared, Denise Rotta: None declared Luca Idolazzi: None declared, Davide Gatti Speakers bureau: Davide Gatti reports personal fees from Abiogen, Amgen, Janssen-Cilag, Mundipharma, outside the submitted work., Maurizio Rossini Speakers bureau: AbbVie, Abiogen, Amgen, BMS, Eli-Lilly, Novartis, Pfizer, Sanofi, Sandoz and UCB, Angelo Fassio Speakers bureau: Angelo Fassio reports personal fees from: Abiogen and Novartis, outside the submitted work.

DOI: 10.1136/annrheumdis-2020-eular.3676

\begin{tabular}{l|l}
\hline THU0480 & EXPERIENCE USING DIFFERENT CRITERIA OF \\
& FIBROMYALIA IN PATIENTS WITH ANKYLOSING \\
& SPONDYLITIS: 1990 AMERICAN COLLEGE OF \\
& RHEUMATOLOGY CLASSIFICATION CRITERIA VS. \\
& NEW
\end{tabular}

I. Shapoval ${ }^{1}$, M. Stanislavchuk ${ }^{1}, \mathrm{H}$. Movchan ${ }^{1} .{ }^{1}$ National Pirogov Memorial Medical University, Vinnytsya, Ukraine, Chair of Internal Medicine $\#^{1}$, Vinnytsya, Ukraine

Background: Fibromyalgia (FM) is a very frequent condition in patients with diseases associated with pain syndrome, such as rheumatoid arthritis (RA), ankylosing spondylitis (AS) and other chronic rheumatic diseases. FM, RA and AS has different clinical characteristics, but can share symptoms such as pain, fatigue and sleep disturbance that leads to delay in appropriation correct diagnosis [1] For today well known many different criteria for FM: 1990 American College of Rheumatology (ACR) classification criteria, modified 2010 ACR diagnostic criteria, 2016 Fibromyalgia Diagnostic Criteria and new AAPT Diagnostic Criteria for 